\title{
Association of Complex Fractionated Electrograms with Atrial Myocardial Thickness and Fibrosis
}

Tae-Min Rhee, MD1; So-Ryoung Lee, MD2; Myung-Jin Cha, MD1; Eue-Keun Choi, MD, PhD'; Seil Oh, MD, PhD, FESC, FHRS 1

${ }^{1}$ Department of Internal Medicine, Seoul National University College of Medicine, Seoul, Korea

${ }^{2}$ Department of Internal Medicine, Soonchunhyang University Hospital Seoul, Seoul, Korea
Received: January 23, 2018

Accepted: March 22, 2018

Correspondence: Seil Oh, MD, PhD, FESC, FHRS

Department of Internal Medicine, Seoul National

University College of Medicine, 101 Daehak-ro,

Jongno-gu, Seoul, 03080, Korea.

FAX: +82-2-762-9662

E-mail: seil@snu.ac.kr

Copyright (c) 2018 The Official Journal of Korean Heart Rhythm Society Editorial Board

\begin{abstract}
Background and Objectives: Although ablation of complex fractionated atrial electrograms (CFAE) in atrial fibrillation (AF) is one of the strategies for atrial substrate modification, the mechanism behind CFAE as an electrophysiological substrate remains unclear. We investigated structural differences between CFAE sites and their matched non-CFAE sites by comparing their histopathologic characteristics in canine AF models.
\end{abstract}

Methods: Atrial electrograms of four dogs were obtained from the epicardial site. AF was induced through burst atrial pacing at 600 bpm for 30 min. CFAE sites were identified during AF according to patterns visualized on the electrograms, and their matched non-CFAE sites were selected in the adjacent region, within $5 \mathrm{~mm}$ of each CFAE site. Tissues were harvested from CFAE sites and their matched nonCFAE sites at various locations in both atria. Histopathologic differences were identified between CFAE and non-CFAE sites.

Results: A total of 24 atrial tissues ( 12 with CFAE, 12 with non-CFAE) were evaluated. The atrial myocardium was significantly thicker at CFAE sites $(1757.5 \pm 560.5 \mu \mathrm{m})$ than at non-CFAE sites $(1279.5 \pm 337.2$ $\mu \mathrm{m})(p=0.036)$. At CFAE sites, it was filled with a significantly larger amount of fibrotic tissue than at non-CFAE sites $(22.8 \pm 6.9 \%$ versus $7.2 \pm 4.7 \%, p<0.001)$. Results were consistent across various tissue locations. The distribution of autonomic nerve innervation was similar between CFAE and non-CFAE sites.

Conclusion: This study provides a better understanding of histological characteristics of CFAE sites, namely a thicker wall and greater amount of fibrosis. These findings may be associated with the development of CFAE and its pathophysiological contribution to AF.

Key Words: - Atrial Fibrillation - Complex Fractionated Electrograms -Myocardium -Radiofrequency Catheter Ablation - Fibrosis 


\section{Introduction}

Although radiofrequency ablation has been an important treatment strategy for persistent atrial fibrillation (AF), it is remains challenging and results are unsatisfactory. ${ }^{1}$ Several additive substrate modification strategies have been attempted, to improve the recurrence rate and long-term outcomes of nonparoxysmal AF. Nademanee et al. reported that complex fractionated atrial electrograms (CFAEs) could be ideal target sites for ablation of refractory AF, and CFAE-guided ablation may show a higher success rate and survival benefit than pulmonary vein isolation alone. ${ }^{2,3}$

However, the pathophysiologic mechanism of CFAE has not yet been clarified, and the structural relationship between CFAE sites and AF is also unknown. Since negative results of CFAE ablation have also been reported, characteristics of CFAE sites as an electrophysiological substrate should be more accurately identified and reflected in clinical practice. ${ }^{4,5}$ Therefore, we sought to evaluate the structural differences between CFAE and non-CFAE sites by comparing their histopathologic features.

\section{Materials and Methods}

\section{Animal Preparation}

The study protocol was approved by the Seoul National University Hospital Institutional Animal Care and Use Committee (approval No. 16-0136-S1A0). A total of four adult mongrel dogs, weighing 20-25 kg, were involved in the present study. Following the standard and approved protocols, all dogs were anesthetized with thiopental $(20 \mathrm{mg} / \mathrm{kg} \mathrm{IV})$ and intubated with cuffed endotracheal tubes for mechanical respiration followed by gaseous anesthesia ( $1-2 \%$ isoflurane $\left./ \mathrm{O}_{2}\right)$. All measures were taken to ensure that discomfort, distress, pain, and injury were limited as much as possible. Standard surface electrocardiogram leads were monitored continuously throughout the entire study, and intermittent arterial blood gas analyses with ventilator adjustments were done to correct for any metabolic abnormalities. An electrical heating pad was used to maintain a body temperature of $36-37^{\circ} \mathrm{C}$. For the electrophysiological study and simultaneous tissue harvesting, a median sternotomy and pericardiectomy were performed to access the canine heart directly. The epicardial patch electrodes for electrophysiological studies were fixed to the atrial free walls by simple sutures.

\section{Electrophysiological Study Protocol}

Atrial electrograms were amplified and filtered from 0.05 to $500 \mathrm{~Hz}$ and were displayed and recorded on a Prucka Cardiolab EP System (GE Medical Systems, Fairfield, CT, USA). Atrial pacing was performed at twice the current threshold. Epicardial bipolar electrograms were recorded using a standard diagnostic catheter with a 2-mm tip electrode, a 1-mm band electrode and a 2-mm distal inter-electrode spacing (Livewire, St. Jude Medical, Minnetonka, MN, USA).

\section{Atrial Fibrillation Induction and CFAE Detection Protocol}

AF was induced with 600-bpm burst atrial pacing for 30 min, and AF that was sustained longer than 10 min was included in the evaluation. The CFAE sites were determined according to the patterns on the electrograms. ${ }^{2}$ Electrograms with the following two characteristics simultaneously were considered a CFAE: 1) atrial electrograms that had fractionated electrograms composed of two deflections or more, and/or perturbation of the baseline with continuous deflection of a prolonged activation complex, over a 10-s recording period; 2) atrial electrograms with a very short cycle length $(\leq 120 \mathrm{~ms})$ averaged over a 10-s recording period. A 10-s duration was used for the assessment of the CFAE to avoid nonspecific sites and to confirm consistent fractionation. The matched non-CFAE sites were selected in the adjacent region within $5 \mathrm{~mm}$ from each CFAE site.

\section{Histopathologic Examination}

Myocardial tissue from the CFAE and non-CFAE sites, detected at various locations of both atria, were harvested. The excised CFAE and non-CFAE site tissues were fixed in $4 \%$ formalin for more than $48 \mathrm{~h}$ for staining and histopathologic 
examination. Sections of the obtained tissues were cut at $2 \mathrm{~mm}$ thickness by a microtome, and serial sections were stained with hematoxylin-eosin ( $\mathrm{HE})$, Masson's trichrome (MT), tyrosine hydroxylase (TH), and choline acetyltransferase (ChAT). Digital photographs of microscopic images were taken under 40-, 100-, and 200-fold magnification (BX51TF; Olympus, Tokyo, Japan), and transmitted to the computer for quantitative analyses using image analysis software (ImageJ, National Institutes of Health, USA). Thickness of the atrial myocardium was measured in sections stained with $\mathrm{HE}$ at 40 -fold magnification. For every section stained by MT, three to five visual fields under 200-fold magnification were randomly chosen to measure the amount of fibrosis in the atrial myocardium and the proportion of the area containing fibrosis was calculated for each section. The distribution of sympathetic or parasympathetic nerve innervation in the atrial myocardium was examined by quantitative analysis of nerve density.

\section{Statistical Analysis}

Continuous variables were expressed as mean \pm standard deviation and were analyzed using the paired $t$ test. All probability values were two-sided, and a $p$-value $<0.05$ was considered statistically significant. Statistical analyses were performed using SPSS statistical package version 19.0 (IBM Corp, Armonk, NY, USA).

\section{Results}

\section{Baseline Distribution of CFAE in Various Atrial Tissues}

A total of 24 atrial myocardial tissues (12 from CFAE sites and 12 from their matched non-CFAE sites) were evaluated. Table 1 shows the anatomical location and distribution of the tissues used in the analysis. Of the 12 sites, 7 were located in the right atrium (the lateral wall proximally anterior to the anterior right ganglionated plexus [RALW], the mid portion of the sulcus terminalis $[\mathrm{ST}]$, and the sinus nodal area $[\mathrm{SN}]$ ), and five in the left atrium (mid portion of the ligament of Marshall [LOM], posterior wall [LAPW], roof area [LAR], and left pulmonary vein antrum $[\mathrm{LPVA}])$.
Table 1. Anatomical distribution of CFAE versus non-CFAE sites of four dogs

\begin{tabular}{lcc}
\hline Location & $\begin{array}{c}\text { CFAE } \\
(\mathrm{n}=12)\end{array}$ & $\begin{array}{c}\text { Non-CFAE } \\
(\mathrm{n}=12)\end{array}$ \\
\hline Right atrium & 2 & 2 \\
RALW & 3 & 3 \\
ST & 2 & 2 \\
SN & & \\
Left atrium & 2 & 2 \\
LOM & 1 & 1 \\
LAPW & 1 & 1 \\
LAR & 1 & 1 \\
LPVA & & \\
\hline
\end{tabular}

CFAE, complex fractionated atrial electrograms; LAPW, left atrial posterior wall; LAR, left atrial roof area; LOM, mid portion of ligament of Marshall; LPVA, left pulmonary vein antrum; RALW, lateral wall just anterior area near the anterior right ganglionated plexus; SN, sinus nodal area; ST, mid portion of the sulcus terminalis

\section{Relationship Between Thickness and Amount of Fibrosis in the Atrial Myocardium According to the CFAE}

Table 2 summarizes the histopathologic differences between CFAE and non-CFAE sites. The atrial myocardium was significantly thicker at CFAE sites $(1757.5 \pm 560.5 \mu \mathrm{m})$ than at matched non-CFAE sites $(1279.5 \pm 337.2 \mu \mathrm{m})(p=0.036)$. The same trend was observed in the subgroups of the left and right atria. In particular, the difference was greatest in the $\mathrm{SN}$ in the right atrium (CFAE site, $1536.5 \pm 114.7 \mu \mathrm{m}$; non-CFAE site, $825.5 \pm 134.3 \mu \mathrm{m}$ ) and RALW (CFAE site, 1935.9 $\pm 539.2 \mu \mathrm{m}$; non-CFAE site, $1409.3 \pm 275.7 \mu \mathrm{m})$. There was a pronounced difference in the amount of fibrosis between CFAE and their matched non-CFAE sites. The atrial myocardium at the CFAE sites was filled with significantly larger amounts of fibrotic tissue than matched non-CFAE sites $(22.8 \pm 6.9 \%$ versus $7.2 \pm 4.7 \%, p<0.001$ ) (Figure 1). The same results were obtained at all locations in the left and right atria. The difference was observed to be particularly large in the LAPW (30.3\% versus $6.6 \%)$. 
Table 2. Histopathologic characteristics of harvested tissues according to the presence of CFAE

\begin{tabular}{lcccccc}
\hline & \multicolumn{3}{c}{ Thickness of atrial myocardium $(\mu \mathrm{m})$} & \multicolumn{3}{c}{ Fibrosis in atrial myocardium $(\%)$} \\
\cline { 2 - 7 } & $\begin{array}{c}\text { CFAE } \\
(\mathrm{n}=12)\end{array}$ & $\begin{array}{c}\text { Non-CFAE } \\
(\mathrm{n}=12)\end{array}$ & $\begin{array}{c}P \text {-value } \\
\text { Total }\end{array}$ & $\begin{array}{c}\text { CFAE } \\
(\mathrm{n}=12)\end{array}$ & $\begin{array}{c}\text { Non-CFAE } \\
(\mathrm{n}=12)\end{array}$ & $P$-value \\
\hline Right atrium & $1757.5 \pm 560.5$ & $1279.5 \pm 337.2$ & 0.036 & $22.8 \pm 6.9$ & $7.2 \pm 4.7$ & $<0.001$ \\
$\quad$ RALW & $1678.6 \pm 599.3$ & $1192.4 \pm 293.9$ & 0.088 & $23.4 \pm 6.5$ & $8.2 \pm 5.8$ & 0.001 \\
ST & $1935.9 \pm 539.2$ & $1409.3 \pm 275.7$ & 0.529 & $22.1 \pm 4.1$ & $6.3 \pm 0.5$ & 0.130 \\
SN & $1621.0 \pm 821.5$ & $1267.4 \pm 204.1$ & 0.498 & $26.5 \pm 7.9$ & $10.1 \pm 8.3$ & 0.061 \\
Left atrium & $1536.5 \pm 114.7$ & $825.5 \pm 134.3$ & 0.012 & $18.5 \pm 2.3$ & $6.5 \pm 1.3$ & 0.134 \\
LOM & $2073.0 \pm 253.7$ & $1627.7 \pm 339.8$ & 0.086 & $21.8 \pm 8.1$ & $5.5 \pm 1.5$ & 0.006 \\
$\quad$ LAPW & $1893.6 \pm 0.0$ & $1387.4 \pm 0.0$ & - & $15.9 \pm 10.7$ & $4.0 \pm 1.0$ & 0.335 \\
$\quad$ LAR & - & $1395.7 \pm 0.0$ & - & $30.3 \pm 0.0$ & $6.6 \pm 0.0$ & - \\
$\quad$ LPVA & $2252.4 \pm 0.0$ & $1868.0 \pm 0.0$ & - & $24.6 \pm 0.0$ & $6.9 \pm 0.0$ & - \\
\hline
\end{tabular}

CFAE, complex fractionated atrial electrograms; LAPW, left atrial posterior wall; LAR, left atrial roof area; LOM, mid portion of ligament of Marshall; LPVA, left pulmonary vein antrum; RALW, lateral wall just anterior area near the anterior right ganglionated plexus; SN, sinus nodal area; ST, mid portion of the sulcus terminalis

A CFAE site: Left atrial roof area

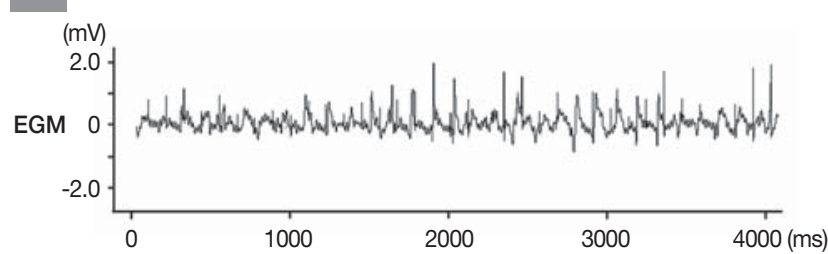

B CFAE site: Left atrial roof area

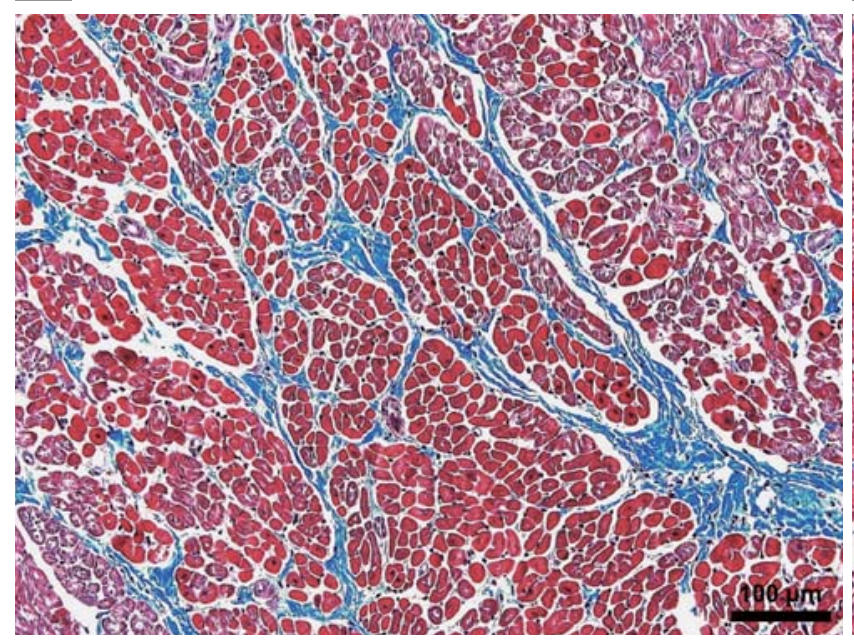

C Non-CFAE site: Bachmann's bundle

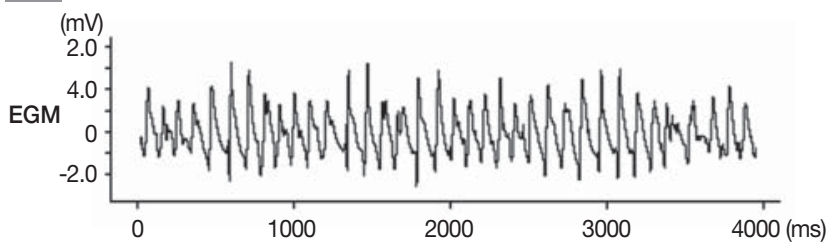

Don-CFAE site: Bachmann's bundle

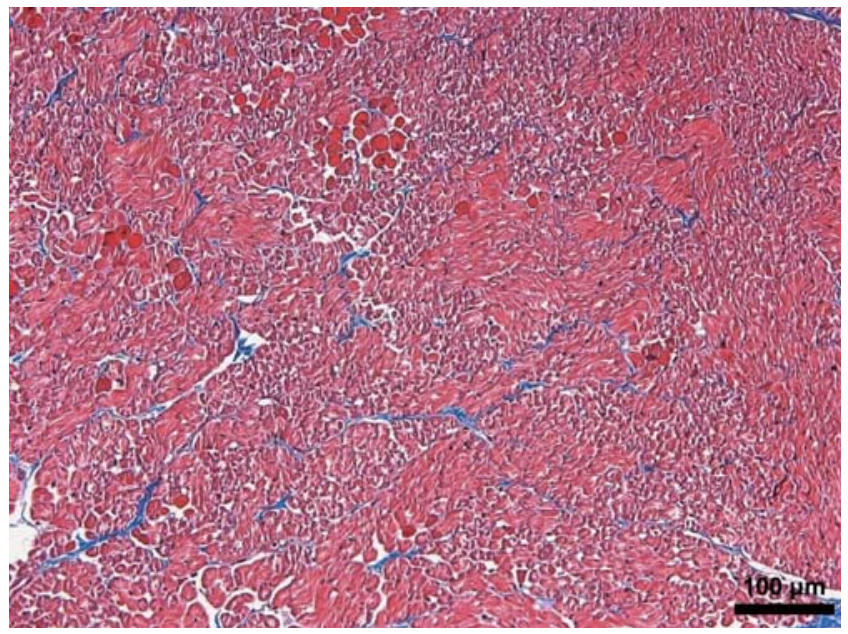

Figure 1. A representative example of CFAE versus non-CFAE site associated with amount of fibrosis in atrial myocardium

Electrograms and section stained by Masson's trichrome (magnification $\times 200$ ) of the CFAE site at left atrial roof area (A and B) are compared to those of the non-CFAE site at Bachmann's bundle ( $C$ and $D)$. The proportion of fibrosis area of the myocardium at CFAE site $(24.5 \%)$ is significantly larger than that of non-CFAE site (6.9\%).

CFAE, complex fractionated atrial electrogram; EGM, electrogram 


\section{Distribution of Autonomic Nerve Innervation}

The distribution of autonomic nerve innervation, which was assessed by the nerve density, did not differ significantly between the CFAE and their matched non-CFAE sites for either the sympathetic (CFAE site, $1128.1 \pm 186.5 \mu \mathrm{m}^{2} / \mathrm{mm}^{2}$; non-CFAE site, $\left.1170.4 \pm 187.1 \mathrm{~mm}^{2} / \mathrm{mm}^{2}, p=0.570\right)$ or parasympathetic nerves (CFAE site, $299.0 \pm 106.4 \mu \mathrm{m}^{2} / \mathrm{mm}^{2}$; non-CFAE site, $\left.373.7 \pm 136.8 \mu \mathrm{m}^{2} / \mathrm{mm}^{2}, p=0.145\right)$, regardless of the location from which the atrial tissue was harvested (Table 3 ).

Table 3. Comparison of autonomic nerve distribution according to the presence of CFAE

\begin{tabular}{|c|c|c|c|c|c|c|}
\hline & \multicolumn{3}{|c|}{ Sympathetic nerve density $\left(\mu \mathrm{m}^{2} / \mathrm{mm}^{2}\right)$} & \multicolumn{3}{|c|}{ Parasympathetic nerve density $\left(\mu \mathrm{m}^{2} / \mathrm{mm}^{2}\right)$} \\
\hline & $\begin{array}{c}\text { CFAE } \\
(n=12)\end{array}$ & $\begin{array}{c}\text { Non-CFAE } \\
(n=12)\end{array}$ & $P$-value & $\begin{array}{c}\text { CFAE } \\
(n=12)\end{array}$ & $\begin{array}{c}\text { Non-CFAE } \\
(n=12)\end{array}$ & $P$-value \\
\hline Total & $1128.1 \pm 186.5$ & $1170.4 \pm 187.1$ & 0.570 & $299.0 \pm 106.4$ & $373.7 \pm 136.8$ & 0.145 \\
\hline Right atrium & $1113.3 \pm 196.7$ & $1125.5 \pm 167.0$ & 0.878 & $295.1 \pm 118.3$ & $369.1 \pm 152.5$ & 0.240 \\
\hline RALW & $1080.5 \pm 63.7$ & $1102.8 \pm 259.2$ & - & $348.1 \pm 135.8$ & $325.9 \pm 86.3$ & - \\
\hline ST & $1038.9 \pm 152.2$ & $1102.8 \pm 77.7$ & - & $219.4 \pm 99.1$ & $366.7 \pm 227.9$ & - \\
\hline SN & $1327.8 \pm 385.0$ & $1216.6 \pm 102.1$ & - & $366.7 \pm 62.9$ & $438.9 \pm 7.9$ & - \\
\hline Left atrium & $1157.8 \pm 181.6$ & $1260.0 \pm 211.5$ & 0.567 & $316.7 \pm 23.6$ & $394.4 \pm 7.9$ & 0.177 \\
\hline LOM & $1194.4 \pm 7.9$ & $1316.6 \pm 23.6$ & - & - & - & - \\
\hline LAPW & $1311.1 \pm 0.0$ & $1200.0 \pm 0.0$ & - & $333.3 \pm 0.0$ & $388.9 \pm 0.0$ & - \\
\hline LAR & $1244.4 \pm 0.0$ & $944.4 \pm 0.0$ & - & - & - & - \\
\hline LPVA & $844.4 \pm 0.0$ & $1522.2 \pm 0.0$ & - & $300.0 \pm 0.0$ & $400.0 \pm 0.0$ & - \\
\hline
\end{tabular}

CFAE, complex fractionated atrial electrograms; LAPW, left atrial posterior wall; LAR, left atrial roof area; LOM, mid portion of ligament of Marshall; LPVA, left pulmonary vein antrum; RALW, lateral wall just anterior area near the anterior right ganglionated plexus; SN, sinus nodal area; ST, mid portion of the sulcus terminalis

\section{Discussion}

\section{Major Findings}

In the present study, CFAE was observed at various locations throughout the left and right atrium. Compared with matched non-CFAE sites, a thicker atrial myocardium and a larger amount of fibrosis was observed at all CFAE sites, in all locations. However, distribution of the autonomic nervous system did not differ significantly between the CFAE sites and the matched nonCFAE sites.

\section{Histopathologic Features of CFAE Sites}

CFAE sites are known to exhibit low voltage and slow conduction. ${ }^{6}$ In addition, focal discharge, wave break and fusion, and pivoting activation observed in CFAE are known to contribute to the maintenance of AF through wave propagation.? CFAE areas could indicate the re-entry of fibrillation waves or the overlap of other wavelets superimposed in the same area at different times. ${ }^{1}$ CFAE sites may also have rotor boundaries or be associated with migrating rotors and wave breaks rather than the center of a stable rotor itself.8 In our previous study, CFAE sites have been identified as AF substrates different from the AF nest.? One computational study suggested that CFAE is more strongly related to the tissue characteristics of the atrial substrate than to the electrical activation system. ${ }^{10}$ In other words, CFAE sites are expected to be associated with the underlying heterogeneous architecture of the myocardium. You et al. reported in their pathological study that the myocardium in CFAE sites was 
heterogeneously arranged and had more intercellular substances. ${ }^{11}$ However, till date, there has been only one study on the histopathologic characteristics of CFAE sites, and this study included only qualitative analysis. In the present study, we have provided a pathological analysis of CFAE sites and included confirmatory results through the quantitative and paired analysis of CFAE sites and matched non-CFAE sites.

First, we presented thicker atrial myocardium as one of the histological features of CFAE. According to the cardiac computed tomography study by Park et al., thickness of the interatrial septum was associated with the extent of the CFAE area and acute procedural success of catheter ablation. ${ }^{12}$ Furthermore, left atrial wall thickness measured by cardiac computed tomography was also found to be more in the CFAE area $(3.0 \pm 1.0 \mathrm{~mm})$ than in the non-CFAE area $(2.2 \pm 0.9 \mathrm{~mm})$, which is consistent with our results. ${ }^{13}$ These results suggest that atrial myocardial thickness is closely related to the CFAE sites. This also suggests the possibility of heterogeneous tissue organization and arrangement at CFAE sites, which may be an important factor associated with AF and the procedural success of ablating atrial substrates.

Second, we found that a large amount of atrial myocardial fibrosis was identified as another histopathologic characteristic of CFAE. It has already been suggested that fibrosis is an important component of the AF substrate in several human and animal AF model studies. ${ }^{14}$ In addition, structural heterogeneities, such as random microstructural alterations, have been reported to be the major pathophysiological mechanisms of CFAE and persistent AF. ${ }^{15}$ Particularly in the CFAE sites, increased fibrosis with decreased connexin 43 has been suggested to underlie structural abnormalities. ${ }^{16}$ Jacquement et al. suggested in their computational microstructural modeling study, that microfibrosis could also contribute to CFAE genesis. ${ }^{17}$ In addition to simple collagen deposition, fibroblast proliferation in the atria has also been shown to affect CFAE generation during AF. ${ }^{18}$ Since this study has confirmed that increased atrial fibrosis is one of the microscopic characteristics of CFAE sites, we may now better understand the underlying mechanism of CFAE-guided AF ablation. However, in studies analyzing late gadolinium enhancement cardiac magnetic resonance imaging of left atrial wall composition, it has been reported that normal substrate without fibrosis is even more associated with CFAE than that with fibrotic sites. ${ }^{19}$ Although inconsistent, it is clear that fibrosis is closely related to the CFAE sites, which should be clarified in future studies.

The autonomic basis for CFAE formation and effects of the autonomic nervous system on CFAE are well known. ${ }^{20}$ However, in terms of autonomic nerve distribution assessed by nerve density, results of the present study did not show any significant difference between CFAE and non-CFAE sites. Since autonomic nerve distribution analysis was limited to nerve density in this study, further work with detailed quantitative analysis including the number and distribution of ganglionated plexus is warranted.

\section{Clinical Implications}

Until now, it has been unclear how CFAE sites function as AF substrates, and the mechanism by which CFAE-targeted AF ablation is helpful for AF termination was unknown. In the present study, the histological features of the CFAE site were described through paired comparisons, but this result alone cannot determine the exact mechanism of CFAE development or the clinical significance of CFAE-targeted ablation. However, it is clear that atrial myocardial thickness and the amount of myocardial fibrosis are closely related to the CFAE sites. Given these features, overlapping of myocardial fiber layers, anisotropy, and epicardial-endocardial dissociation may more likely occur at the CFAE sites. ${ }^{13}$ In this regard, we can still utilize CFAE as a possible substrate-modification target for AF ablation.

However, the results of clinical studies on CFAE-targeted AF ablation are inconsistent. Nademanee et al. have repeatedly presented clinical benefits of CFAE-targeted ablation of AF through their series of studies. ${ }^{2,3,21}$ Contradictory to this, it has been reported in STAR AF II, BOCA, and CHASE AF trials that adding CFAE ablation to pulmonary vein isolation had no additional benefit. ${ }^{22-24}$ Results of meta-analyses till date are also controversial. ${ }^{4,25}$ Further studies on the efficacy of CFAE-targeted AF ablation as well as human studies on the pathological characteristics of CFAE sites are warranted.

\section{Study Limitations}

Since the present study is a preclinical animal study, the results 
of this study are difficult to apply directly to clinical practice. First, inducing AF in normal healthy dogs may be different from clinical AF in human patients. Second, since all of the electrograms were acquired from epicardial electrodes, conducting endocardial mapping may show results that are different from this experiment. Third, because CFAE sites change continuously, interpretation of the results should take into account the recording duration and spatiotemporal stability of the electrograms. Fourth, in some CFAE sites, such as LAPW and LPVA, accurate measurement of wall thickness was difficult owing to limitations of the harvested tissue and was therefore excluded from the analysis. Lastly, when defining CFAE in this study, we used the criteria proposed by Nademanee et al. ${ }^{2}$ However, Rostock et al. defined CFAE as fractional potentials exhibiting more than two deflections from the isoelectric line and/or potentials with continuous electrical activity without an isoelectric line. ${ }^{26}$ On the other hand, Oral et al. used a definition of electrograms with a cycle length of $120 \mathrm{~ms}$ or less than in the coronary sinus, or showing fractionated or continuous electric activity. ${ }^{27}$ Since the definition of CFAE is not uniform, the results of the present study cannot be extrapolated to all CFAE-related studies.

\section{Conclusions}

A thicker atrial myocardium and a larger amount of fibrosis were identified as the most important histopathologic characteristics of CFAE sites compared to their matched nonCFAE sites. These results may help elucidate the underlying mechanism of CFAE and the implications for catheter ablation.

\section{Acknowledgments}

The researchers Moo Kang Kim and Ji Yoon Shin were responsible for the tissue sampling, processing, and slide production. Dr. Jee-Soo Lee assisted in slide observation, taking photography and image processing.

\section{Disclosure}

This study was supported by grant no. 2016-1169 from the Seoul National University Hospital Research Fund.

\section{References}

1) Aksu T, Guler TE, Yalin K, Oto A. Unanswered questions in complex fractionated atrial electrogram ablation. Pacing Clin Electrophysiol. 2016;39:1269-1278.

2) Nademanee K, McKenzie J, Kosar E, Schwab M, Sunsaneewitayakul B, Vasavakul T, Khunnawat C, Ngarmukos T. A new approach for catheter ablation of atrial fibrillation: mapping of the electrophysiologic substrate. J Am Coll Cardiol. 2004;43:2044-2053.

3) Nademanee K, Schwab MC, Kosar EM, Karwecki M, Moran MD, Visessook N, Michael AD, Ngarmukos T. Clinical outcomes of catheter substrate ablation for high-risk patients with atrial fibrillation. J Am Coll Cardiol. 2008;51:843-849.

4) Fadahunsi O, Talabi T, Olowoyeye A, Iluyomade A, Shogbesan O, Donato A. Ablation of complex fractionated atrial electrograms for atrial fibrillation rhythm control: a systematic review and metaanalysis. Can J Cardiol. 2016;32:791-802.

5) Scott PA, Silberbauer J, Murgatroyd FD. The impact of adjunctive complex fractionated atrial electrogram ablation and linear lesions on outcomes in persistent atrial fibrillation: a meta-analysis. Europace. 2016;18:359-367.

6. Park JH, Pak HN, Kim SK, Jang JK, Choi JI, Lim HE, Hwang C, Kim YH. Electrophysiologic characteristics of complex fractionated atrial electrograms in patients with atrial fibrillation. J Cardiovasc Electrophysiol. 2009;20:266-272.

7) Yamabe H, Morihisa K, Tanaka Y, Uemura T, Enomoto K, Kawano $\mathrm{H}$, Ogawa $\mathrm{H}$. Mechanisms of the maintenance of atrial fibrillation: role of the complex fractionated atrial electrogram assessed by noncontact mapping. Heart Rhythm. 2009;6:1120-1128.

8) Kalifa J, Tanaka K, Zaitsev AV, Warren M, Vaidyanathan R, Auerbach D, Pandit S, Vikstrom KL, Ploutz-Snyder R, Talkachou A, Atienza F, Guiraudon G, Jalife J, Berenfeld O. Mechanisms of wave fractionation at boundaries of high-frequency excitation in the posterior left atrium of the isolated sheep heart during atrial fibrillation. Circulation. 2006;113:626-633.

9) Oh S, Kong HJ, Choi EK, Kim HC, Choi YS. Complex fractionated electrograms and AF nests in vagally mediated atrial fibrillation. Pacing Clin Electrophysiol. 2010;33:1497-1503.

10) Varela M, Aslanidi OV. Role of atrial tissue substrate and electrical activation pattern in fractionation of atrial electrograms: a computational study. Conf Proc IEEE Eng Med Biol Soc. 2014;2014:1587-1590. 
11) You DJ, Chang D, Zhang SL, Yang DH, Gao LJ, Yin XM, Chu ZL, Xia YL, Wang YC, Dong YX, Yang YZ. Substrate of complex fractionated atrial electrograms: evidence by pathologic analysis. Chin Med J (Engl). 2012;125:4393-4397.

12) Park YM, Park HC, Ban JE, Choi JI, Lim HE, Park SW, Kim YH. Interatrial septal thickness is associated with the extent of left atrial complex fractionated atrial electrograms and acute procedural outcome in patients with persistent atrial fibrillation. Europace. 2015;17:1700-1707.

13) Wi J, Lee HJ, Uhm JS, Kim JY, Pak HN, Lee M, Kim YJ, Joung B. Complex fractionated atrial electrograms related to left atrial wall thickness. J Cardiovasc Electrophysiol. 2014;25:1141-1149.

14) Boldt A, Wetzel U, Lauschke J, Weigl J, Gummert J, Hindricks G, Kottkamp H, Dhein S. Fibrosis in left atrial tissue of patients with atrial fibrillation with and without underlying mitral valve disease. Heart. 2004;90:400-405.

15) Haissaguerre M, Shah AJ, Cochet H, Hocini M, Dubois R, Efimov I, Vigmond E, Bernus O, Trayanova N. Intermittent drivers anchoring to structural heterogeneities as a major pathophysiological mechanism of human persistent atrial fibrillation. J Physiol. 2016;594:2387-2398.

16) Liu X, Shi HF, Tan HW, Wang XH, Zhou L, Gu JN. Decreased connexin 43 and increased fibrosis in atrial regions susceptible to complex fractionated atrial electrograms. Cardiology. 2009; 114:22-29.

17) Jacquemet V, Henriquez CS. Genesis of complex fractionated atrial electrograms in zones of slow conduction: a computer model of microfibrosis. Heart Rhythm. 2009;6:803-810.

18) Ashihara T, Haraguchi R, Nakazawa $K$, Namba T, Ikeda T, Nakazawa Y, Ozawa T, Ito M, Horie M, Trayanova NA. The role of fibroblasts in complex fractionated electrograms during persistent/permanent atrial fibrillation: implications for electrogram-based catheter ablation. Circ Res. 2012;110:275-284.

19) Jadidi AS, Cochet H, Shah AJ, Kim SJ, Duncan E, Miyazaki S, Sermesant M, Lehrmann H, Lederlin M, Linton N, Forclaz A, Nault I, Rivard L, Wright M, Liu X, Scherr D, Wilton SB, Roten L, Pascale P, Derval N, Sacher F, Knecht S, Keyl C, Hocini M, Montaudon M, Laurent F, Haissaguerre M, Jais P. Inverse relationship between fractionated electrograms and atrial fibrosis in persistent atrial fibrillation: combined magnetic resonance imaging and high-density mapping. J Am Coll Cardiol. 2013;62:802-812.
20) Chaldoupi SM, Linnenbank AC, Wittkampf FH, Boldt LH, H VANW, VJ VAND, Doevendans PA, Hauer RN, JM DEB, Loh P. Complex fractionated electrograms in the right atrial free wall and the superior/posterior wall of the left atrium are affected by activity of the autonomic nervous system. J Cardiovasc Electrophysiol. 2012;23:26-33.

21) Nademanee K, Amnueypol M, Lee F, Drew CM, Suwannasri W, Schwab MC, Veerakul G. Benefits and risks of catheter ablation in elderly patients with atrial fibrillation. Heart Rhythm. 2015;12:4451.

22) Verma A, Jiang CY, Betts TR, Chen J, Deisenhofer I, Mantovan R, Macle L, Morillo CA, Haverkamp W, Weerasooriya R, Albenque JP, Nardi S, Menardi E, Novak P, Sanders P. Approaches to catheter ablation for persistent atrial fibrillation. $N$ Engl J Med. 2015;372:1812-1822

23) Wong KC, Paisey JR, Sopher M, Balasubramaniam R, Jones M, Qureshi N, Hayes CR, Ginks MR, Rajappan K, Bashir Y, Betts TR. No Benefit of Complex Fractionated Atrial Electrogram Ablation in Addition to Circumferential Pulmonary Vein Ablation and Linear Ablation: Benefit of Complex Ablation Study. Circ Arrhythm Electrophysiol. 2015;8:1316-1324.

24) Vogler J, Willems S, Sultan A, Schreiber D, Luker J, Servatius H, Schaffer B, Moser J, Hoffmann BA, Steven D. Pulmonary vein isolation versus defragmentation: The CHASE-AF clinical trial. $J$ Am Coll Cardiol. 2015;66:2743-2752.

25) Afzal MR, Samanta A, Chatta J, Ansari B, Atherton S, Sabzwari S, Turagam M, Lakkireddy D, Houmsse M. Adjunctive ablation strategies improve the efficacy of pulmonary vein isolation in nonparoxysmal atrial fibrillation: a systematic review and metaanalysis. Expert Rev Cardiovasc Ther. 2017;15:227-235.

26) Rostock T, Rotter M, Sanders P, Takahashi Y, Jais P, Hocini M, Hsu LF, Sacher F, Clementy J, Haissaguerre M. High-density activation mapping of fractionated electrograms in the atria of patients with paroxysmal atrial fibrillation. Heart Rhythm. 2006;3:27-34

27) Oral H, Chugh A, Good E, Wimmer A, Dey S, Gadeela N, Sankaran S, Crawford T, Sarrazin JF, Kuhne M, Chalfoun N, Wells D, Frederick M, Fortino J, Benloucif-Moore S, Jongnarangsin K, Pelosi F, Jr., Bogun F, Morady F. Radiofrequency catheter ablation of chronic atrial fibrillation guided by complex electrograms. Circulation. 2007;115:2606-2612. 